\title{
Reduction of Respiratory Motion During PET/CT by Pulsatile-Flow Ventilation: A First Clinical Evaluation
}

\author{
John O. Prior*1, Nicolas Péguret*2, Anastasia Pomoni ${ }^{1}$, Martin Pappon ${ }^{1}$, Michele Zeverino $^{3}$, Bastien Belmondo ${ }^{4}$, \\ Alban Lovis ${ }^{5}$, Mahmut Ozsahin ${ }^{2}$, Monique Vienne ${ }^{6}$, and Jean Bourhis ${ }^{2}$ \\ ${ }^{1}$ Department of Nuclear Medicine and Molecular Imaging, Lausanne University Hospital, Lausanne, Switzerland; ${ }^{2}$ Department of \\ Radiation Oncology, Lausanne University Hospital, Lausanne, Switzerland; ${ }^{3}$ Department of Medical Physics, Lausanne University \\ Hospital, Lausanne, Switzerland; ${ }^{4}$ Department of Physiotherapy, Lausanne University Hospital, Lausanne, Switzerland; ${ }^{5}$ Department \\ of Pneumology, Lausanne University Hospital, Lausanne, Switzerland; and ${ }^{6}$ BIRD Institute of Pulmonary Care, Villeneuve-Loubet, \\ France
}

Respiratory motion negatively affects PET/CT image quality and quantitation. A novel Pulsatile-Flow Ventilation (PFV) system reducing respiratory motion was applied in spontaneously breathing patients to induce sustained apnea during PET/CT. Methods: Four patients (aged $65 \pm 14 \mathrm{y}$ ) underwent PET/CT for pulmonary nodule staging (mean, $11 \pm 7 \mathrm{~mm}$; range, $5-18 \mathrm{~mm}$ ) at $63 \pm 3 \mathrm{~min}$ after ${ }^{18} \mathrm{~F}-\mathrm{FDG}$ injection and then at $47 \pm 7$ min afterward, during PFV-induced apnea (with imaging lasting $\geq 8.5 \mathrm{~min}$ ). Anterior-posterior thoracic amplitude, SUV $V_{\text {max }}$, and $S U V_{\text {peak }}\left(S U V_{\text {mean }}\right.$ in a 1-cm-diameter sphere) were compared. Results: PFV PET/CT reduced thoracic amplitude (80\%), increased mean lesion $\operatorname{SUV}_{\max }(29 \%)$ and $\operatorname{SUV}_{\text {peak }}(11 \%)$, decreased lung background SUV peak (25\%), improved lesion detectability, and increased $S U V_{\text {peak }}$ lesion-to-background ratio (54\%). On linear regressions, SUV $\max$ and $S_{\text {SUV }}$ peak significantly improved (by $35 \%$ and $23 \%$, respectively; $P \leq 0.02$ ). Conclusion: PFV-induced apnea reduces thoracic organ motion and increases lesion SUV, detectability, and delineation, thus potentially affecting patient management by improving diagnosis, prognostication, monitoring, and external-radiation therapy planning.

Key Words: PET/CT; High-Frequency Percussive Ventilation; HFPV; respiratory motion; Pulsatile-Flow Ventilation

J Nucl Med 2016; 57:416-419

DOI: 10.2967/jnumed.115.163386

$\mathbf{P}$

ET/CT has become a major oncologic imaging modality for diagnosis, prognostication, therapy monitoring, and radiation therapy planning (1). Respiratory motion has significant negative effects on image quality, on the accuracy with which PET quantifies lesion activity, on fusion accuracy, and on delineation of lesion volume (2). Several advanced PET/CT respiration-gating techniques have been developed to palliate thoracic organ motion (2). All have intrinsic limitations, such as longer PET acquisitions, mixing of several tissue positions in a single bin, and difficulty

Received Jul. 5, 2015; revision accepted Nov. 16, 2015.

For correspondence or reprints contact: Jean Bourhis, Lausanne University Hospital, Bugnon 46, 1011 Lausanne, Switzerland.

E-mail: jean.bourhis@chuv.ch

${ }^{*}$ Contributed equally to this work.

Published online Dec. 3, 2015.

COPYRIGHT (c) 2016 by the Society of Nuclear Medicine and Molecular Imaging, Inc. with irregular breathing patterns. In addition, not only PET but also CT should be gated to avoid introducing supplementary attenuation correction and quantification errors (2). Today, these techniques are not universally applied and no consensus exists as to the best method to compensate for respiratory motion.

HFPV (high-frequency percussive ventilation; OxyCare $\mathrm{GmbH}$ ) was first designed to promote airway clearance through a percussive flow of air. On the basis of this hygienic effect, HFPV was then developed in inpatient burn units, with percussive airflow favoring evacuation of airway debris secondary to inhalation injury (3). The physical principle is to deliver high-frequency ventilation $(>400 / \mathrm{min})$ in low ventilation cycles (10-30 cycles/min). Clinical experience demonstrated improved lung compliance, oxygenation, and ventilation compared with conventional ventilation in intensive care. These benefits extended the indication as a salvage modality for acute respiratory distress syndrome, with clinical evidence supported by smaller trials. Successive subtidal breathing with added high-frequency oscillations to both the inhalation phase and the exhalation phase facilitates oxygen diffusion and carbon dioxide removal. We refined this technique to obtain apnealike respiratory motion stabilization at full inspiration for medical imaging and radiation therapy (4). We achieved apnea lasting 8-16 min, allowing radiation therapy in nonintubated patients (4).

We aimed at establishing Pulsatile-Flow Ventilation (The BIRD Institute of Pulmonary Care, Villeneuve-Loubet, France) PET/CT (PFV) to suppress respiratory motion and quantify lesion detectability and quantification.

\section{MATERIALS AND METHODS}

\section{Patient Population}

Four patients (mean age $\pm \mathrm{SD}, 65 \pm 14 \mathrm{y}$ ) with a pulmonary tumoral lesion (mean size, $11 \pm 7 \mathrm{~mm}$; range, 5-18 $\mathrm{mm}$ ) deemed treatable by radiation therapy were assessed in this research protocol, which was authorized by the State of Vaud Ethics Committee on Human Research. All patients signed an informed consent form. We had originally enrolled 5 patients (between November 2014 and April 2015), but 1 patient refused to undergo PFV PET/CT. The clinical characteristics of the 4 assessed patients are presented in Table 1 .

\section{${ }^{18}$ F-FDG PET/CT Acquisition}

Images were acquired on a time-of-flight PET/CT scanner (Discovery D690; GE Healthcare) with scatter and point-spread-function recovery corrections, $60 \mathrm{~min}$ after injection of ${ }^{18} \mathrm{~F}-\mathrm{FDG}(3.5 \mathrm{MBq} / \mathrm{kg})$ and after the patients had fasted for at least $6 \mathrm{~h}$ (PET: $2 \mathrm{~min} / \mathrm{bed}$ position, 47 slices, $256 \times 256$ matrix, ordered-subsets expectation 
TABLE 1

Clinical Characteristics of Patients

\begin{tabular}{lcccc}
\hline \multicolumn{1}{c}{ Characteristic } & Patient 1 & Patient 2 & Patient 3 & Patient 4 \\
\hline Age $(\mathrm{y})$ & 70 & 79 & 65 & 45 \\
Sex & $\mathrm{M}$ & $\mathrm{M}$ & $\mathrm{F}$ & $\mathrm{F}$ \\
Weight $(\mathrm{kg})$ & 95 & 66 & 47 & 66 \\
Body mass index $\left(\mathrm{kg} / \mathrm{m}^{2}\right)$ & 33 & 21 & 18 & 24 \\
Lesion size $(\mathrm{mm})$ & $18 \times 17 \times 15$ & $7 \times 4 \times 6$ & Right lower lobe & Right upper lobe \\
Localization & Left upper lobe & Right upper lobe & NSCLC cT2cNOcM0 & NSCLC pT1aNOM0 \\
Pathologic type & NSCLC pT1NOMO & NSCLC pT1aNOMO & Staging & Stereotactic body radiation \\
PET/CT & Staging & & Staging
\end{tabular}

NSCLC $=$ Non-small cell lung cancer.

maximization, 2 iterations $\times 24 \mathrm{~s}$; CT: $140 \mathrm{kV}, 80-200$ SmartmA, 0.5 s/rotation, 3.75-mm thickness). After the PFV apparatus had been installed, PFV PET/CT was performed with a thorax-dedicated (3-bed) acquisition using the same parameters as for PET/CT. Anterior-posterior chest displacement was monitored with Real-Time Position Management, a respiratory gating system supplied by Varian Medical Systems.

\section{PFV PET/CT}

The HFPV technique (3) was performed using a Transrespirator (International Medical Assistance by Percussionaire). The technique was refined by us to suppress low-frequency cycles in order to induce sustained apnealike chest stabilization in spontaneously breathing patients and thus maintain adequate partial pressures of oxygen and carbon dioxide without respiratory motion. We previously found the technique capable of suppressing normal respiratory motion in volunteers (11.6-min median time), allowing us to perform a successful clinical feasibility study in radiation therapy ( $n=3$ patients) (4). Patients were conscious, nonsedated, and nonintubated, with a mouthpiece interface for delivering noninvasive high-frequency ventilation.

\section{Image Analysis}

We used an Advantage workstation (version 4.6, GE Healthcare) and Osirix, version 6.5.2 (Pixmeo), to compute SUV, $\mathrm{SUV}_{\text {max }}, \mathrm{SUV}_{\text {peak }}$ (mean SUV per 1-cm sphere), lesion $\mathrm{SUV}_{\text {mean }}$ using a $42 \% \mathrm{SUV}_{\max }$-segmentation threshold $\left(\mathrm{SUV}_{\text {mean }}{ }_{42} \%\right)$, corresponding lesion volume (volume ${ }_{42} \%$ ), and total-lesion glycolysis $\left(\mathrm{TLG}_{42} \%=\mathrm{SUV}_{\text {mean } 42 \%} \times\right.$ volume $\left._{42} \%\right)$.

\section{Statistical Analysis}

Wilcoxon matched-pairs signed-rank tests were used for means comparisons, and linear regression was used for assessing PFV PET/CT changes. The statistical software was Stata 13.1 (StataCorp), and the significance level was $P<0.05$.

\section{RESULTS}

PFV PET/CT could be successfully performed for at least $8.5 \mathrm{~min}$ in all patients. The patients did not find the procedure uncomfortable, thanks to two prior 5-min training sessions performed outside the PET/CT scanner on the same day $(n=1)$ or a different day $(n=3)$. In all patients, oxygen saturation remained normal (95\%-100\% under a $100 \%$ fraction of inspired oxygen) with intrinsic breathing motion suppressed and the diaphragm stopped in the maximal inspiratory position. The anterior-posterior thoracic amplitude greatly diminished, with a trend toward statistical significance despite the low number of patients (Table 1; Fig. 1A and Supplemental Fig. 1; supplemental materials are available at http://jnm.snmjournals.org).
PET/CT SUV metrics (Table 2) showed a trend toward a $29 \%$ higher mean $\mathrm{SUV}_{\text {max }}$ (Fig. 1C) and a $25 \%$ lower $\mathrm{SUV}_{\text {peak }}$ in lung parenchyma, leading to a $54 \%$ better lesion-to-lung SUV peak $_{\text {ratio }}$ (Fig. 1D). Linear regression analysis showed that HPV-induced apnea significantly increased $\mathrm{SUV}_{\max }$ by $35 \%(P=0.033)$ and $\mathrm{SUV}_{\text {peak }}$ by $23 \%(P=0.006)$ (Fig. $\left.1 \mathrm{~B}\right)$.

PET image quality was much improved, with reduced blurring, increased lesion SUV, and decreased lung parenchyma activity. For instance, in patient 1 the pulmonary lesion was not seen on maximumintensity-projection imaging (although ${ }^{18} \mathrm{~F}$-FDG uptake was increased in multiplanar reconstructions) (Fig. 2A) but became clearly distinguishable on PFV PET/CT (Fig. 2B).

The effect of lesion blurring is seen in patient 2, in whom two adjacent pulmonary lesions had different ${ }^{18} \mathrm{~F}$-FDG uptake characteristics (Figs. 3A and 3B and Supplemental Fig. 3). PFV PET/CT led to higher SUVs on the craniocaudal profile (Fig. 3C).
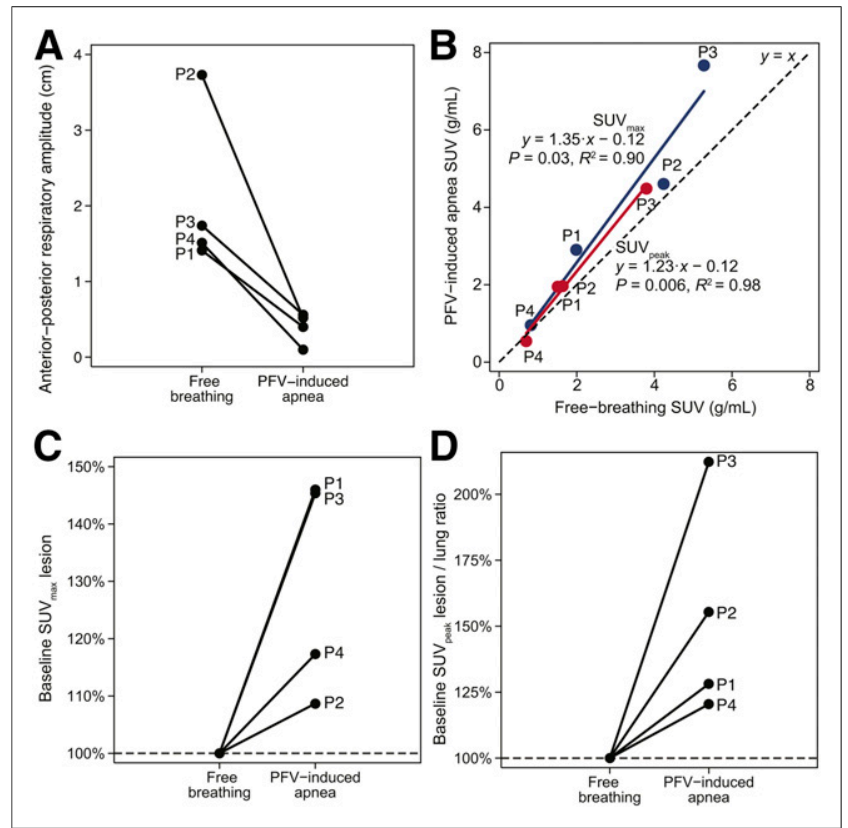

FIGURE 1. Effect of PFV-induced apnea compared with free breathing on anterior-posterior respiratory motion (A), PET/CT uptake (B), relative increase in $S U V_{\max }(C)$, and lesion-to-lung ratio $S_{U V} V_{\text {peak }}(D)$. 
TABLE 2

${ }^{18}$ F-FDG PET/CT Uptake Parameters

\begin{tabular}{|c|c|c|c|c|}
\hline Variable & Free breathing & PFV PET/CT & Difference & $P$ \\
\hline Anterior-posterior motion (mm) & $21 \pm 11$ & $4 \pm 2$ & $-80 \% \pm 12 \%$ & 0.07 \\
\hline $\mathrm{SUV}_{\max }(\mathrm{g} / \mathrm{mL})$ & $3.1 \pm 1.0$ & $4.0 \pm 1.4$ & $+29 \% \pm 12 \%$ & 0.07 \\
\hline $\operatorname{SUV}_{\text {peak }}(\mathrm{g} / \mathrm{mL})$ & $1.9 \pm 1.3$ & $2.2 \pm 1.6$ & $+11 \% \pm 23 \%$ & 0.5 \\
\hline $\mathrm{SUV}_{\text {mean } 42 \%}(\mathrm{~g} / \mathrm{mL})$ & $1.9 \pm 0.5$ & $2.4 \pm 1.6$ & $+22 \% \pm 24 \%$ & 0.14 \\
\hline Metabolic volume $_{42 \%}(\mathrm{~mL})$ & $2.6 \pm 2.4$ & $1.6 \pm 1.4$ & $-19 \% \pm 34 \%$ & 0.6 \\
\hline $\mathrm{TLG}_{42 \%}(\mathrm{~g})$ & $5.8 \pm 7.6$ & $3.9 \pm 3.5$ & $-5 \% \pm 39 \%$ & 0.99 \\
\hline SUV $V_{\text {peak }}$ (lung) $(\mathrm{g} / \mathrm{mL})$ & $0.52 \pm 0.05$ & $0.39 \pm 0.14$ & $-25 \% \pm 19 \%$ & 0.14 \\
\hline Lesion-to-lung SUV $_{\text {peak }}$ ratio & $3.8 \pm 2.9$ & $6.8 \pm 6.9$ & $+54 \% \pm 41 \%$ & 0.07 \\
\hline SUV $_{\max }$ (patient 2: mediastinal lymph nodes, $\left.n=5\right)(\mathrm{g} / \mathrm{mL})$ & $2.7 \pm 0.5$ & $3.3 \pm 0.78$ & $+23 \% \pm 14 \%$ & 0.04 \\
\hline
\end{tabular}

In patient 3 (Figs. 4A and 4B), the pulmonary lesion had a large SUV increase whereas the SUV of lung parenchyma decreased because of hyperinflation, leading to the highest increase in $S U V_{\text {peak }}$ lesion-to-background ratio (Fig. 1D). The $\mathrm{SUV}_{\max }$ of ${ }^{18}$ F-FDGpositive mediastinal lymph nodes (Supplemental Fig. 4) was visibly increased on PFV PET/CT (Table 2), and there was improved PET contrast in small structures (esophagus, stomach, ribs, aortic wall) (Fig. 4 and Supplemental Fig. 4).

Patient 4 had a small, $0.5-\mathrm{mm}$, pulmonary lesion not visible on PET images under free-breathing conditions (Supplemental Fig. 5). PFV PET/CT rendered it fairly distinguishable on multiplanar reconstructions (17\% $\mathrm{SUV}_{\max }$ increase, $22 \%$ decrease in neighboring lung parenchyma), although it remained invisible on the maximumintensity-projection image.

\section{DISCUSSION}

PFV-induced apnea was clinically feasible for at least $8.5 \mathrm{~min}$ in nonintubated, nonsedated patients, allowing full-thorax PET acquisitions with decreased respiratory motion, increased lesion $\mathrm{SUV}_{\max }$ and $\mathrm{SUV}_{\text {peak }}$, and improved image quality.

Several studies using time-based methods or a deep-inspiration breath-hold have shown $\mathrm{SUV}_{\max }$ gain (21\%-69\% and 10\%-23\% between gated and nongated acquisitions, respectively) (2). For instance, Garcia Vicente et al. (5) found a major increase in $\mathrm{SUV}_{\max }$

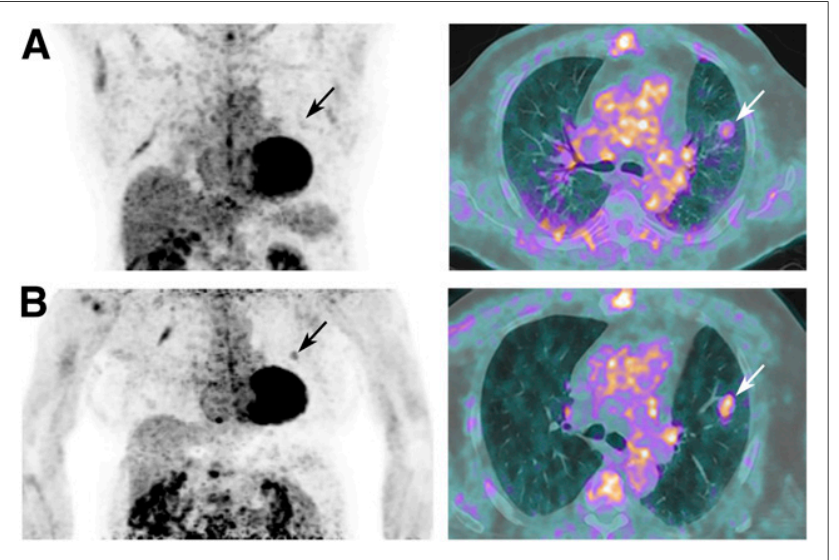

FIGURE 2. Patient 1: Pulmonary nodule (arrows) in free breathing (A) and PFV-induced apnea (B). Nodule is visible only on maximum intensity projection image in apnea. More images are available online (Supplemental Fig. 2).
$(83 \%)$ in their 42-patient population. The patients had nodules measuring $1.2 \pm 0.56 \mathrm{~cm}$, and the study used an older scanner without time-of-flight or point-spread-function recovery, potentially explaining the relatively larger difference found in their study than in ours. Many studies have compared mean difference in $\mathrm{SUV}_{\max }$ due to respiratory gating, but only Werner et al. presented results from linear regression between gated and nongated $\operatorname{SUV}_{\max }(6)$, finding an $11 \%$ increase due to gating $(y=1.119 x+1.54)$. Our study led to a larger increase (35\%), showing much promise for increased PET activity recovery.

In a phantom study, Bowen et al. showed that respiratory gating allowed recovery of only $60 \%$ of the true motion-free $\mathrm{SUV}_{\max }$ tumorto-background ratio, which corresponded to a $14 \%$ increase compared with free-moving acquisitions (tumor-to-background ratio, $9.0 \rightarrow 10.3$ ), and there was another $10 \%$ increase when motion was totally suppressed (tumor-to-background ratio, $10.3 \rightarrow 11.2$, a $24 \%$ increase from free-moving) (7). Thus, comparing our results with other respirationgated studies may not reveal the true potential of the method.

Although breath-hold techniques have been developed with satisfactory results, maintaining an apnea duration of more than $30 \mathrm{~s}$ is difficult for sick patients. Our PFV PET/CT is a noninvasive technique that can successfully achieve an apnea period of at least $8.5 \mathrm{~min}$ in most patients, allowing larger fields of exploration (e.g., 3 bed positions).

Obviously, our feasibility study had several limitations. First, the limited number of patients and consequent limited spectrum of lesion size and location did not allow the full potential of the technique to be assessed. However, all patients had improvements in image quality, lesion detectability, and SUV quantitation. Additionally, the PET visibility of several structures was strikingly improved (ribs, aortic walls, stomach walls), and these encouraging preliminary results should lead to further clinical trials in larger patient cohorts.

Second, it is possible that the additional time required to set up the PFV apparatus (47 $\pm 7 \mathrm{~min}$ ) favorably affected ${ }^{18} \mathrm{~F}-\mathrm{FDG}$ lesion uptake, thus contributing to the SUV increase. Indeed, Tahari et al. demonstrated that delayed acquisition could increase standardized uptake value adjusted for lean body mass (SUL) by $34 \%$ alone (8), but their delayed time was longer ( $80 \mathrm{~min}$ ). In fact, respiratory gating improved SUL by only another $17 \%$. It is hard to believe that in our study most of the $\mathrm{SUV}_{\max }$ improvement would be due to additional uptake time. For instance, in patient 3 , the centrally positioned subcarinal lymph node showed a minimal change in $\operatorname{SUV}_{\text {max }}(2.5 \rightarrow 2.6$, a $4 \%$ gain) whereas the mediastinal lymph nodes, which have greater motion, showed a greater change $(2.2 \rightarrow 3.0$, a $35 \%$ gain $)$. 


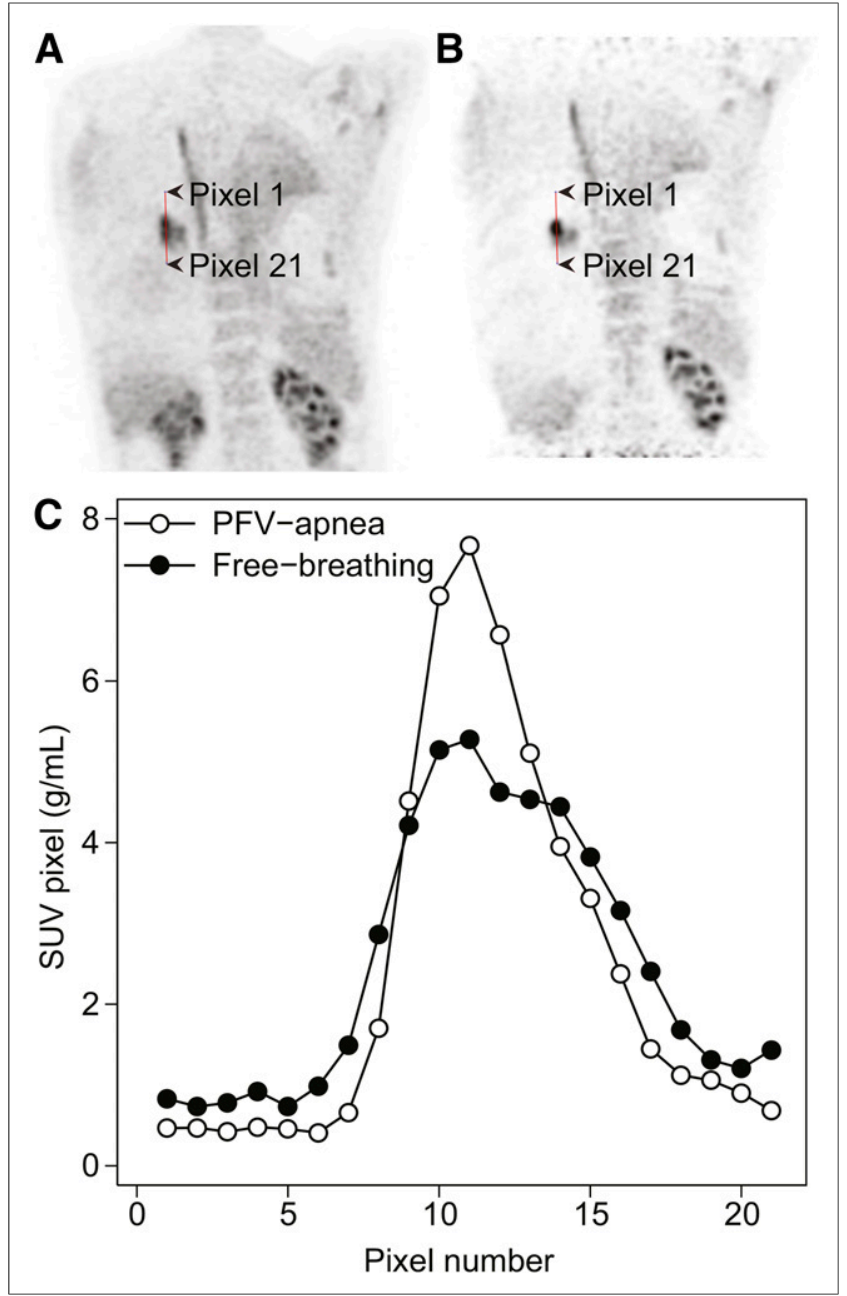

FIGURE 3. Patient 2: Coronal 2-cm-thick PET slice through tumor in free breathing (A) and PFV-induced apnea (B), with corresponding SUV pixel values $(C)$ along vertical line through tumor (arrowheads). SUV is higher and blurring and SUV lower in lung parenchyma on PFV-induced apnea image. More images are available online (Supplemental Fig. 3).

Further PFV PET/CT applications can be envisioned, such as for other lesions affected by respiratory motion (breast, chest wall, liver, pancreas, bile duct and gallbladder, esophagus and stomach, spleen, heart, kidneys), for radiation oncology therapy planning

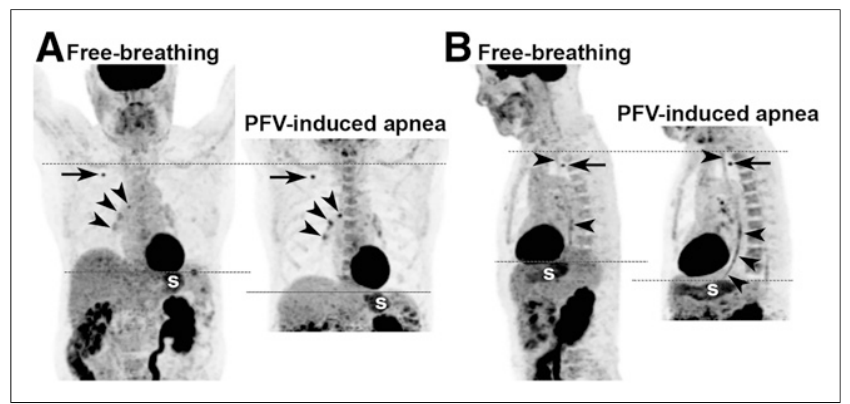

FIGURE 4. Patient 3: Maximum-intensity projection with ${ }^{18} \mathrm{~F}-\mathrm{FDG}-$ positive pulmonary lesion (arrows) and mediastinal lymph nodes (arrowheads in A) during free breathing (A) and PFV-induced apnea (B). Lesionto-background ratio and visibility of esophagus (arrowheads in B) are better on PFV-induced apnea image. $s=$ stomach.
(9), or for image-guided biopsies in interventional radiology. Studies investigating the ability of PFV PET/CT to discriminate between malignant and nonmalignant lesions would be desirable, as would studies using radiopharmaceuticals other than ${ }^{18} \mathrm{~F}-\mathrm{FDG}$. This method would also be directly applicable to PET/MR hybrid imaging, as MR is also influenced by respiratory motion.

Finally, the clinical value of PFV PET/CT needs to be established in comparison with existing respiration-gating techniques and delayed acquisition in larger patient cohorts. PFV PET/CT shares the same principle as percussion-assisted radiation therapy, and our center has already demonstrated the feasibility of using PFV PET/CT to plan radiation therapy (4).

\section{CONCLUSION}

PFV successfully induced at least $8.5 \mathrm{~min}$ of apnealike suppressed respiratory motion during PET/CT, with a resulting improvement in PET image quality, SUV quantitation, and lesion volume delineation. These promising results call for studies on larger cohorts to establish the clinical value of PFV PET/CT.

\section{DISCLOSURE}

The costs of publication of this article were defrayed in part by the payment of page charges. Therefore, and solely to indicate this fact, this article is hereby marked "advertisement" in accordance with 18 USC section 1734 . This work benefited from a collaborative grant between Lausanne University Hospital (Lausanne, Switzerland), IMAP (Villeneuve-Loubet, France), and Ablatech (Toulouse, France). Monique Vienne is an employee of IMAP, which distributes the Transrespirator used in this study, but she had no control over the data. No other potential conflict of interest relevant to this article was reported.

\section{ACKNOWLEDGMENTS}

We thank Eric Bider for adapting the PFV apparatus for PET/CT, and also our physiotherapists, Kathleen Grant, Julien Simons, and Olivier Long.

\section{REFERENCES}

1. Usmanij EA, de Geus-Oei LF, Bussink J, Oyen WJ. Update on F-18-fluoro-deoxyglucose-PET/computed tomography in nonsmall cell lung cancer. Curr Opin Pulm Med. 2015;21:314-321.

2. Pépin A, Daouk J, Bailly P, Hapdey S, Meyer ME. Management of respiratory motion in PET/computed tomography: the state of the art. Nucl Med Commun. 2014;35:113-122.

3. Allan PF, Osborn EC, Chung KK, Wanek SM. High-frequency percussive ventilation revisited. J Burn Care Res. 2010;31:510-520.

4. Péguret N, Ozsahin M, Zeverino M, et al. Apnea-like suppression of respiratory motion: first evaluation in radiotherapy. Radiother Oncol. In press.

5. García Vicente AM, Soriano Castrejon AM, Talavera Rubio MP, et al. ${ }^{18}$ F-FDG PET-CT respiratory gating in characterization of pulmonary lesions: approximation towards clinical indications. Ann Nucl Med. 2010;24:207-214.

6. Werner MK, Parker JA, Kolodny GM, English JR, Palmer MR. Respiratory gating enhances imaging of pulmonary nodules and measurement of tracer uptake in FDG PET/CT. AJR. 2009;193:1640-1645.

7. Bowen SR, Nyflot MJ, Herrmann C, et al. Imaging and dosimetric errors in 4D PET/CT-guided radiotherapy from patient-specific respiratory patterns: a dynamic motion phantom end-to-end study. Phys Med Biol. 2015;60:3731-3746.

8. Tahari AK, Lodge MA, Wahl RL. Respiratory-gated PET/CT versus delayed images for the quantitative evaluation of lower pulmonary and hepatic lesions. J Med Imaging Radiat Oncol. 2014;58:277-282.

9. Guerra L, Meregalli S, Zorz A, et al. Comparative evaluation of CT-based and respiratory-gated PET/CT-based planning target volume (PTV) in the definition of radiation treatment planning in lung cancer: preliminary results. Eur J Nucl Med Mol Imaging. 2014;41:702-710. 